\title{
Подход к моделированию функиионирования взаимосвязанных систем энергетики в условиях возмущений и его программная поддержка
}

\author{
A.В. Еделев ${ }^{1}$, א.т.н., старший научный сотрудник, flower@isem.irk.ru \\ H.M. Береснева ${ }^{1}$, к.т.н., научный сотрудник, beresneva@isem.irk.ru \\ ${ }^{1}$ Институт систем энергетики им. А.А. Мелентьева СО РАН, \\ 2. Иркутск, 664033, Россия
}

Целью исследования является разработка подхода к моделированию функционирования взаимосвязанных систем энергетики в условиях возмущений, который имеет универсальный характер по отношению к различным классам возмущений и энергетическим комплексам разных уровней территориальной иерархии.

Актуальность работы определяется необходимостью обеспечения устойчивого функционирования энергетики в условиях различных возмущений - от высоких колебаний цен на энергоносители и ухудшения погодных условий до крупномасштабных природных катаклизмов и техногенных катастроф.

Новизна подхода по сравнению с другими заключается в его ориентации на применение высокопроизводительных вычислений для ускорения расчетов и в явном разделении модели энергетической инфраструктуры на структурную и функциональную части.

Структурная часть представляет топологию инфраструктуры в виде сети, функциональная описывает распределение потоков энергоресурсов по инфраструктуре и ее реакцию на воздействие возмущения. Деление модели энергетической инфраструктуры на структурную и функциональную части используется как основополагающий принцип при моделировании не только систем энергетики, но и энергетических комплексов, которые в предлагаемом подходе рассматриваются как метасистемы взаимосвязанных систем энергетики.

Модель энергетического комплекса строится путем объединения структурных и функциональных частей моделей отдельных систем энергетики на основе предопределенных взаимосвязей между ними. Универсальный характер предлагаемого подхода также поддерживается его программной реализацией с помощью средств метапрограммирования языка $\mathrm{C}++$, что значительно экономит время при создании пакетов прикладных программ, предназначенных для оценки адаптивности энергетических комплексов.

Применение подхода продемонстрировано на разработке модели энергетики страны, которая используется в распределенном пакете прикладных программ для глобального или пространственного анализа уязвимости, поиска критических элементов энергетического комплекса.

Ключевые слова: система энергетики, энергетический комплекс, адаптивность, уязвимость, распределенная вычислительная среда, пакет прикладных программ.

Критически важной для устойчивого развития экономики инфраструктурой является энергетический комплекс (ЭК) любого уровня территориальной иерархии. Поэтому не теряют своей актуальности исследования энергетики, касающиеся обеспечения устойчивого функционирования ее отраслевых систем в условиях различных возмущений, начиная с больших колебаний цен на энергоносители, ухудшения погодных условий, напрямую влияющих на работу возобновляемых источников энергии, заканчивая крупномасштабными природными катаклизмами (наводнения, землетрясения, ураганы). Эти исследования направлены на оценку возможностей адаптации систем энергетики (СЭ) к возмущениям.
Адаптивность оценивается с позиций степеней проявления ущерба (оценка недопоставок топлива), снижения технологических возможностей отраслевых объектов, критичности реализованных возмущений для СЭ, а также эффективности примененных компенсирующих мероприятий.

Если с небольшими возмущениями система может справиться сама, то компенсация воздействия крупных возмущений и их последствий возможна лишь в случае дополнительных технологических и организационных мероприятий. Во время крупного возмущения реконфигурируется сеть СЭ, подключаются резервные источники и накопители, хранилища, перераспределяются имеющиеся ресурсы и 
сбрасывается нагрузка для приспособления системы к возмущению и его последствиям. По окончании возмущения происходит возврат СЭ в нормальное или близкое к нему состояние путем обратной реконфигурации сети, перезапуска основных источников и восстановления поврежденных объектов. Далее составляются планы по повышению адаптивности СЭ, под которой понимается способность системы прогнозировать возникновение возмущений, готовиться противостоять им, восстанавливаться после их воздействия [1].

Уязвимость отражает масштаб и величину последствий возмущения для системы в целом. Анализ уязвимости выполняется в период поглощения системой возмущений, когда критичность их последствий для нее максимальна. Полученные оценки позволяют выявить недостатки структуры системы, например, наиболее уязвимые для данного класса возмущений инфраструктурные объекты [2, 3]. Полученная в этом случае информация о возможном ущербе, о наличии доступа к наиболее уязвимым инфраструктурным объектам, об имеющихся технических и финансовых резервах является отправной точкой для планирования повышения адаптивности СЭ [4-7].

Независимо от размера возмущения и уровня территориальной иерархии важно учитывать, что СЭ в рамках ЭК тесно связаны друг с другом и зачастую развитие возмущений имеет лавинообразный характер. Это означает, что небольшое возмущение, возникшее в одной из частей какой-либо СЭ, может достаточно быстро (в зависимости от комбинации условий внешней среды и инерционности процессов конкретных СЭ) превратиться в крупное, распространившись по взаимосвязям и охватив соседние системы целиком. Таким образом, необходима некая общая концепция, описывающая основные принципы моделирования функционирования компонент взаимосвязанных СЭ, другими словами, инфраструктурных объектов ЭК, в неблагоприятных условиях внешней среды. Такая концепция должна быть применима к возмущениям различных классов и ЭК всех уровней территориальной иерархии.

Была проведена предварительная работа по сравнению подобных концепций [8] и обоснован выбор обобщенного подхода к моделированию взаимосвязанных технических систем [9] как наиболее подходящей концепции. Этот подход использовался для анализа уязвимости электроэнергетической системы Швеции [10] и сетей железных дорог этой страны [11]. В данной статье он используется как основа для разработки универсального подхода к моделированию взаимосвязанных СЭ при воздействии возмущений, обеспечивая необходимый уровень поддержки интеграции моделей с различной степенью детализации отраслевых схем, объединяя существующие в системах механизмы компенсации воздействия возмущений и их последствий. В разработанном подходе интеграция моделей СЭ осуществляется на топологическом уровне, также поддерживается возможность многошагового моделирования воздействия возмущений на ЭК. Автоматизация заявленного подхода реализована в рамках описанного в конце статьи распределенного пакета прикладных программ для анализа уязвимости ЭК страны.

\section{Подход к моделированию взаимосвязанных систем энергетики в условиях возмущений}

Результаты сравнительного анализа, проведенного в [8], показывают, что большинство подходов к моделированию взаимосвязанных критических инфраструктур могут работать лишь с выборочными вариантами их функционирования, тогда как обобщенный подход [9] обеспечивает возможность полного перебора всех возможных комбинаций вариантов функционирования систем и неблагоприятных условий внешней среды, число которых может быть чрезвычайно большим. В этой связи представленный далее подход к моделированию функционирования взаимосвязанных СЭ базируется на применении высокопроизводительных вычислений, что позволяет решать задачу перебора сочетаний вариантов функционирования систем и сценариев возмущений за приемлемое время.

Обобщенный подход к моделированию взаимосвязанных технических систем построен на принципе «система систем» [9]. Интеграция этих систем обеспечивается на топологическом уровне, достигается за счет разбиения модели каждой из систем на взаимосвязанные структурную и функциональную (технологическую) составляющие. Итог такой идеологии моделирования систем - возможность структурной компоновки территориально-распределенных систем любой технологической сложности, обеспечение внутри- и межотраслевого взаимодействия объектов этих систем, на функциональном уровне поддержка их технологических возможностей при изменении конфигураций отраслевых схем. 
Такое преимущество сделало данный подход актуальным и для случая моделирования функционирования СЭ, взаимодействующих в рамках ЭК. Последний в этом случае позиционируется как метасистема критических инфраструктур энергетики (ее отраслевых систем), обладающая значительной территориальной и технологической сложностью, характеризуемая взаимодействием топливных отраслей с электро- и теплоэнергетикой, приводящим к существенным межсистемным сбоям в функционировании отраслей при случайных или преднамеренных возмущениях. Адаптация обобщенного подхода применительно к ЭК позволяет моделировать любой набор отраслевых систем с необходимой степенью детализации объектов и их технологических процессов, имитировать и анализировать одиночные и множественные возмущения в рамках многочисленных вычислительных экспериментов.

Подход к моделированию функционирования компонент взаимосвязанных СЭ или инфраструктурных объектов ЭК в условиях возмущений является адаптацией обобщенного подхода [9] и состоит из следующих положений.

Сначала модель каждой отдельной СЭ разбивается на структурную и функциональную составляющие. Структурная составляющая описывает топологию моделируемой системы в виде сети отраслевых объектов (источников, объектов хранения и транспортировки энергоресурсов, потребителей), имеющих определенную географическую привязку. Функциональная составляющая включает технологическое поведение объектов, описывает распределение потока ресурсов по системе, ее реакцию на воздействие возмущения.

Структурные модели отдельных СЭ объединяются в обобщенную структурную модель ЭК с помощью предопределенных взаимосвязей между системами. В обобщенной структуре идентифицируются функциональные, пространственные и гибридные связи. Функциональные связи образуются в случае зависимости функции объекта одной системы от функции объекта другой системы. Пространственная связь определяется, если два (или более) объекта функционально не зависят друг от друга и расположены в одной местности. Гибридные взаимосвязи отражают функциональную зависимость объектов и территориально привязаны к одной местности.

Возмущения в модели реализуются в виде сценариев удаления объектов отраслевых си- стем из обобщенной структурной модели ЭК и изменения функциональных параметров объектов систем (коэффициентов их производительности, пределов использования ресурсов). После внесения изменений обновляются связи между системами, осуществляется перерасчет потоков в функциональных моделях. Затем анализируются результаты потокораспределения по всему множеству проведенных расчетов. При этом схема анализа, как и характер задания возмущений, зависят от вида проводимого анализа уязвимости (глобальный или пространственный анализ, поиск критических элементов), каждый из которых - самостоятельный инструмент исследования системной уязвимости. Схемы проведения упомянутых видов анализа уязвимости подробно описаны в [12], их предназначение и содержательная интерпретация заключаются в следующем [13].

Глобальный анализ уязвимости [11, 12], направленный на получение общей информации о влиянии возмущений на работоспособность системы в целом, проводится путем моделирования серии возмущений с постепенно увеличивающейся степенью воздействия на систему. Его использование позволяет выявить зависимость падения производительности системы в целом от интенсивности отключений элементов в ней. Результат глобального анализа - график падения производительности системы, не базе которого можно сравнить устойчивость различных отраслевых схем конкретной системы.

Пространственный анализ уязвимости, сфокусированный на поиске критических географических районов, используется для анализа масштабных пространственно-распределенных возмущений (например, природных бедствий) $[11,14,15]$. Он позволяет оценить интенсивность проявления возмущений в различных районах «возмущаемой» географической зоны, выявить наиболее уязвимые с территориальной точки зрения отраслевые объекты. Результат анализа - карта влияния территориальных возмущений внутри достаточно большой географической зоны.

Поиск критических элементов $[11,16]$ ориентирован на определение отраслевых объектов, одиночный или групповой отказ которых вызывает наибольшее снижение работоспособности взаимосвязанных систем. Цель анализа обнаружение всех, даже неожиданных, наборов элементов, критичных для энергетики территориальных объединений. Ключевым показателем критичности отраслевого объекта 
в данном случае является комплексный критерий значимости элемента, формируемый на базе складывающихся на территориях недопоставок энергоресурсов по всему множеству отключений данного объекта. При этом дополнительно могут учитываться приоритетность кратности отключений элементов, критичность недопоставок каждого из ресурсов. Результат данного вида анализа уязвимости - ранжированные списки критичных для территорий отраслевых объектов, перечень наиболее уязвимых для реализованных отключений территориальных объединений.

Моделирование возмущений реализовано в рамках общего для рассмотренных видов анализа уязвимости алгоритма, представленного на рисунке 1. В нем на каждом шаге сценария после воздействия возмущения выполняется расчет на функциональных моделях тех систем, структура которых изменилась благодаря отказу (удалению) или восстановлению (добавлению) элементов сети. Затем в итеративном цикле моделируется распространение возмущения по взаимосвязанным системам. В цикле вызов функциональных моделей выполняется для систем, у которых произошло изменение входящих межсистемных связей. После этого происходит обновление состояния их исходящих межсистемных связей. Условием завершения цикла является отсутствие дополнительных изменений межсистемных связей, что подразумевает прекращение каскадных аварий в моделируемой системе. На последнем этапе для каждой из систем оцениваются последствия воздействия возмущения, после чего происходит переход к новому шагу сценария.

Представленные положения делают разработанный подход к моделированию взаимосвязанных СЭ достаточно универсальным по отношению к различным классам возмущений и ЭК разных уровней территориальной иерархии.

\section{Модель топливно-энергетического комплекса страны для анализа уязвимости}

Далее описывается применение разработанного подхода к моделированию функционирования компонент взаимосвязанных СЭ в условиях возмущений для создания модели энергетики страны, которая затем используется в пакетах прикладных программ, реализующих различные виды анализа уязвимости ЭК.

Построение любой модели СЭ для отдельных отраслевых или общеэнергетических ис- следований $[17,18]$, как правило, подчинено так называемой TRV-идеологии, ключевыми элементами которой являются территории (R), технологии отраслевых объектов (T), производимые или используемые ими энергоресурсы (V). В случае многопериодности дополнительно учитывается временной аспект моделирования, позволяющий имитировать этапность активизации возмущений и подключений механизмов структурной избыточности, этапность воздействия возмущений на отраслевые объекты, особенности функционирования последних в условиях нештатных ситуаций. Поэтому для оценки уязвимости взаимосвязанных СЭ была формализована многопериодная мо-

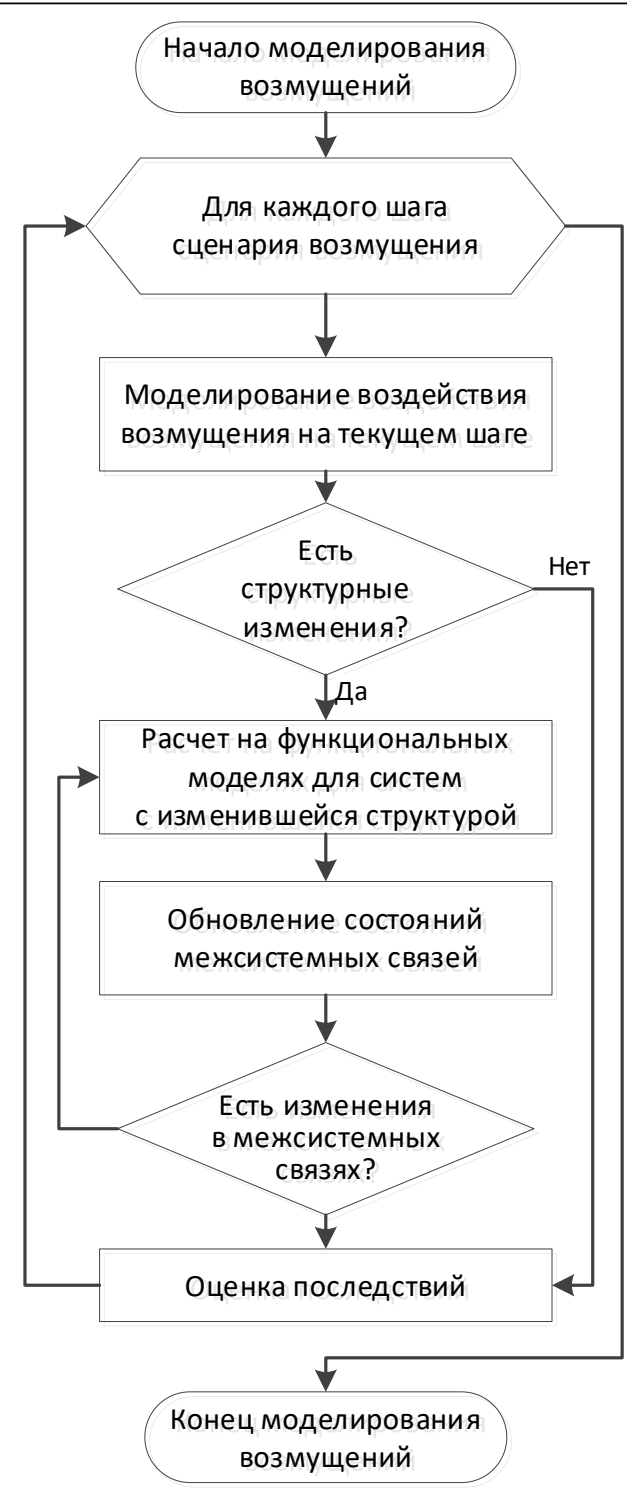

Рис. 1. Алгоритм моделирования возмущеений

Fig. 1. The algorithm for modeling disturbances 
дель ЭК (рис. 2) со следующими характерными особенностями [19].

Модель ЭК поддерживает заданное административно-территориальное деление страны на районы, допускает агрегированное представление в районе производства, потребления и хранения энергоресурсов. Модель может не учитывать территориальное размещение потребителей внутри района, схему внутрирайонных транспортных потоков.

Структурная часть модели ЭК, показанная в левой части рисунка 2 , получается путем объединения структурных моделей отдельных СЭ. Дуги в виде пунктирных линий отражают связи функционального типа между различными СЭ и в основном представляют процессы преобразования энергоресурсов из одного вида в другой. Взаимосвязь отраслей в ЭК поддерживается главным образом на уровне тепловых электрических станций и котельных, работа которых обеспечивается поставками топливных отраслей.

Структурная модель любой СЭ состоит из узлов и дуг. Узлы модели соответствуют территориальным единицам страны и крупным объектам энергетики, имеют географические координаты. Узел-район может быть одновременно потребителем, источником и хранили- щем. Дуги (обозначены сплошными линиями) описывают магистральный транспорт энергоресурсов, выполняя условия баланса производства и потребления (с учетом межрайонного транспорта) отдельного вида энергоресурсов в каждом рассматриваемом районе и по стране в целом.

Если шаг $k$ сценария возмущений разбить на $T_{k}$ периодов и через $v_{t}$ обозначить воздействие возмущения в определенный период времени $t=1, \ldots, T_{k}$, то объединенная функциональная часть модели ЭК может быть описана в виде задачи линейного программирования:

$$
\begin{aligned}
& \sum_{t=1}^{T_{k}}\left[\left(c x_{t}+b s_{t}\right)+p\left(r_{t}-y_{t}\right)+h u_{t} \rightarrow \mathrm{min}\right], \\
& s_{t-1}+A_{t}\left(v_{t}\right) x_{t}+Q_{t} u_{t}-y_{t}-s_{t} \geq 0, \\
& x_{t} \leq D_{t}\left(v_{t}\right), \\
& y_{t} \leq R_{t} \\
& u_{t} \leq U_{t} \\
& s_{t} \leq S_{t}\left(v_{t}\right), \\
& s_{0}=S_{0},
\end{aligned}
$$

где $x_{t}-$ искомый вектор, элементы которого характеризуют интенсивность применения технологических способов функционирования элементов СЭ (добычи, переработки, преобра-

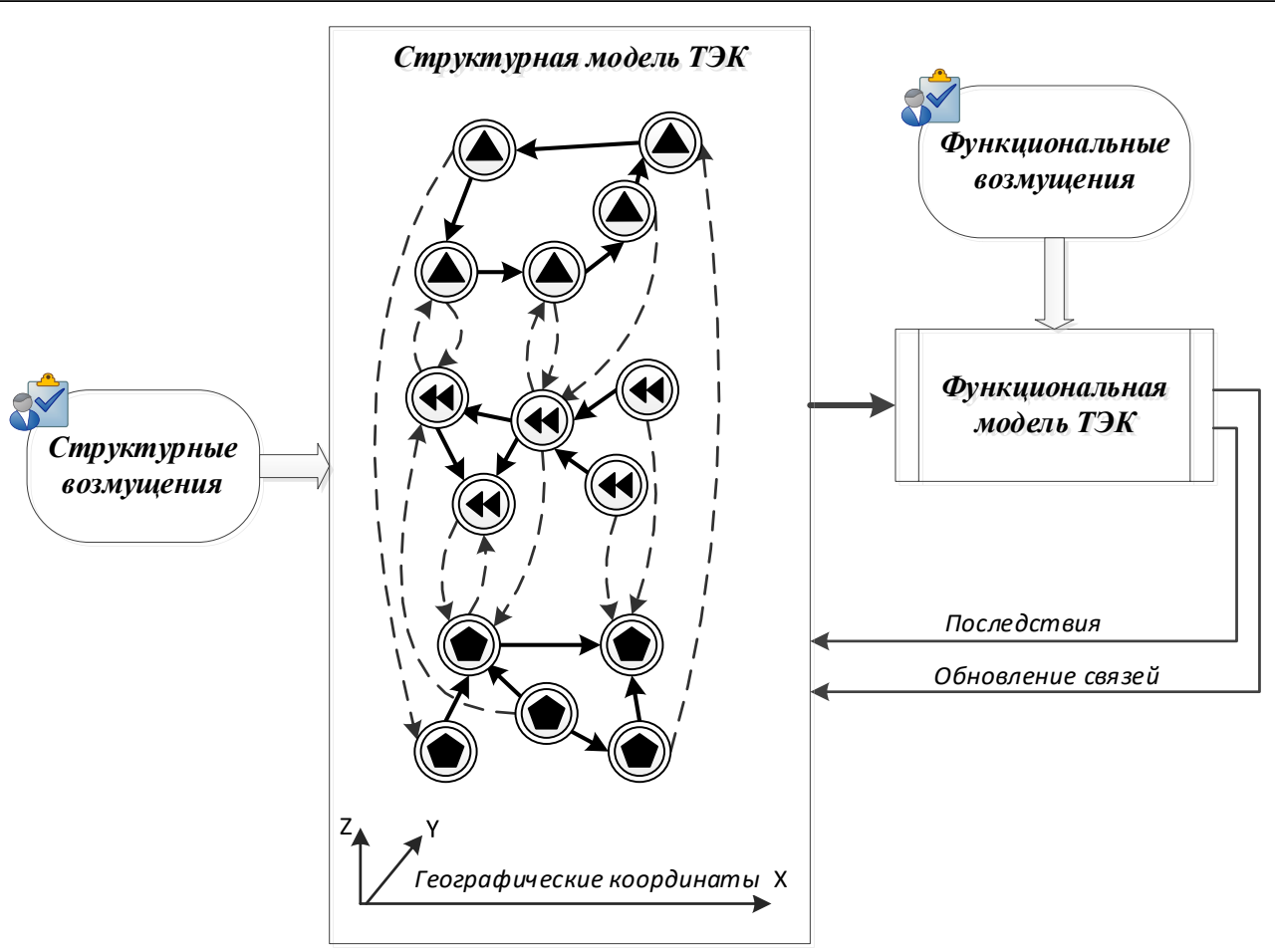

Рис. 2. Моделирование ЭК страны при воздействии крупных возмущений

Fig. 2. Modeling the energy complex of the country affected by large disturbances 
зования и транспорта энергоресурсов) в период времени $t ; y_{t}-$ искомый вектор, элементы которого характеризуют объемы потребления отдельных видов энергоресурсов в период времени $t ; u_{t}$ - искомый вектор, описывающий интенсивность проведения мероприятий по повышению адаптивности в период времени $t$; $s_{t}-$ искомый вектор, компоненты которого характеризуют объемы запасов топлива в период времени $t ; A_{t}$ - матрица, описывающая технологии производства и передачи энергоресурсов в период времени $t$, значения элементов которой зависят от воздействия возмущения $v_{t} ; D_{t}$ вектор, определяющий технически возможные интенсивности применения отдельных технологических и производственных способов в период времени $t$, значения элементов которого зависят от воздействия возмущения $v_{t} ; r_{t}-$ вектор, элементы которого демонстрируют потребности в отдельных видах энергоресурсов в период времени $t ; Q_{t}$ - матрица, отражающая локализацию проведения мероприятий по повышению адаптивности в период времени $t$; $U_{t}-$ вектор, задающий пределы интенсивности проведения мероприятий по повышению адаптивности в период времени $t ; S_{t}$ - вектор, определяющий емкость хранилищ в период времени $t$, значения элементов которого зависят от воздействия возмущения $v_{t} ; c-$ вектор, элементы которого определяют удельные затраты по каждому технологическому способу функционирования элементов СЭ; $b$ - вектор удельных затрат на эксплуатацию хранилищ; $p$ - вектор удельных ущербов, возникающих вследствие недопоставки отдельных видов энергоресурсов потребителям; $h$ - вектор, задающий удельные затраты на подготовку и проведение мероприятий по повышению адаптивности.

Целевая функция (1) является сверткой трех критериев. Первый критерий отражает издержки, связанные с функционированием ЭК, второй - оценивает ущерб от дефицита энергоресурсов из-за воздействия возмущения $v_{t}$, третий - характеризует затраты на подготовку и проведение мероприятий по повышению адаптивности.

Воздействие возмущения $v_{t}$ реализуется элементами матрицы $A_{t}$ и векторов $D_{t}, S_{t}$ в уравнениях (2), (3) и (6) соответственно. Их элементы характеризуют степень деформации различных компонентов СЭ вследствие воздействия возмущения в период времени $t$.

Уровень необходимого снабжения потребителей отдельными видами энергоресурсов задается уравнением (4). Технические ограниче- ния на проведение мероприятий по повышению адаптивности определяются в (5).

Объем запасов топлива в хранилищах в период времени $t$ ограничен их доступной емкостью согласно неравенству (6). Уравнение (7) предполагает, что в начале шага $k$ сценария возмущения (в период времени $t=0$ ) все хранилища имеют некоторый начальный запас энергоресурсов, описываемый вектором $S_{0}$.

В анализе уязвимости преобладают детерминированные подходы, где используются минимаксные критерии и по результатам расчетов на модели, подобной вышеописанной, выбирается такой вариант функционирования инфраструктурных объектов ЭК, для которого максимально возможные последствия после воздействия возмущения являются минимальными [8].

\section{Распределенный пакет прикладных программ для поиска критических элементов ЭК}

Подход к моделированию функционирования компонент взаимосвязанных СЭ (инфраструктурных объектов ЭК) в условиях возмущений был реализован в виде библиотеки шаблонов классов и алгоритмов на языке программирования $\mathrm{C}++$. На основе библиотеки написаны шаблонные классы, представляющие глобальный или пространственный анализ уязвимости, поиск критических элементов. В свою очередь, эти классы-шаблоны являются каркасом основных модулей пакетов прикладных программ, реализующих указанные виды анализа уязвимости ЭК. Для построения готового пакета прикладных программ требуется лишь создать соответствующие классы-обертки, отвечающие за формирование моделей СЭ из исходных данных и осуществляющие вызов функциональных частей моделей, подставить имена этих классов в качестве параметров в каркасы модулей и выполнить сборку пакета. Таким образом, универсальный характер предлагаемого подхода к моделированию взаимосвязанных СЭ в условиях возмущений также поддерживается на уровне его реализации с использованием средств метапрограммирования языка $\mathrm{C}++$, что значительно экономит время при создании пакетов прикладных программ, предназначенных для оценки адаптивности ЭК.

Например, в пакете для поиска критических элементов ЭК можно выделить основные программные модули: 
- формирования списка инфраструктурных объектов ЭК $\left(\mathrm{M}_{1}\right)$;

- создания сценариев возмущений $\left(\mathrm{M}_{2}\right)$;

- реализации алгоритма моделирования возмущений $\left(\mathrm{M}_{3}\right)$;

- поддержки экспертного анализа уязвимости ЭК $\left(\mathrm{M}_{4}\right)$.

Информационно-логические связи объектов предметной области пакета представлены на рисунке 3 в виде двудольного ориентированного графа. Модули $\mathrm{M}_{1}, \mathrm{M}_{2}, \mathrm{M}_{3}, \mathrm{M}_{4}$ представлены серыми овалами, а входные и выходные параметры - окружностями.

Основной задачей модуля $\mathrm{M}_{1}$ является чтение исходной информации Д о территориально-производственной структуре ЭК из БД и формирование на ее основе списка инфраструктурных объектов Э, являющихся потенциальными критическими элементами.

Модуль $\mathrm{M}_{2}$ генерирует $K$ сценариев возмущений $\mathrm{C}_{0}, \mathrm{C}_{1}, \mathrm{C}_{2}, \ldots, \mathrm{C}_{K}$, которые в данном виде анализа уязвимости являются группами элементов определенной кратности из списка Э.

Модуль $\mathrm{M}_{3}$ реализует алгоритм моделирования возмущения, показанный на рисунке 1. В поиске критических элементов воздействие возмущения означает удаление элементов, принадлежащих одному из сценариев $\left(\mathrm{C}_{0}, \mathrm{C}_{1}\right.$, $\mathrm{C}_{2}, \ldots, \mathrm{C}_{K}$ ) структурной части модели ЭК. За- тем $\mathrm{M}_{3}$ согласно алгоритму производит на функциональной части модели ЭК расчет последствий возмущения и выбор оптимальных мероприятий по повышению адаптивности, которые вместе образуют результаты расчета $\Pi_{0}$, $\Pi_{1}, \Pi_{2}, \ldots, \Pi_{K}$, соответствующие сценариям возмущений $\mathrm{C}_{0}, \mathrm{C}_{1}, \mathrm{C}_{2}, \ldots, \mathrm{C}_{K}$.

Так как число $K$ может достигать очень больших значений, поиск критических элементов базируется на применении высокопроизводительных вычислений, что позволяет решить данную задачу за приемлемое время. Это означает, что обработка сценариев возмущений $\mathrm{C}_{0}$, $\mathrm{C}_{1}, \mathrm{C}_{2}, \ldots, \mathrm{C}_{K}$ ведется параллельно, а сами сценарии возмущения $\mathrm{C}_{0}, \mathrm{C}_{1}, \mathrm{C}_{2}, \ldots, \mathrm{C}_{K}$ и соответствующие результаты расчета $\Pi_{0}, \Pi_{1}, \Pi_{2}, \ldots, \Pi_{K}$ размещаются в кластерном хранилище данных Apache Ignite [20].

Анализ результатов расчетов проводится с помощью модуля $M_{4}$, который осуществляет распределенную обработку массива $\Pi_{0}, \Pi_{1}, \Pi_{2}$, $\ldots, \Pi_{K}$, выполняя сформированные исследователем запросы к Apache Ignite.

На выходе пакета получается список критических инфраструктурных объектов А, групповой или одиночный отказ которых вызывает наиболее серьезный ущерб для ЭК.

Сборка, тестирование пакетов прикладных программ и вычислительные эксперименты

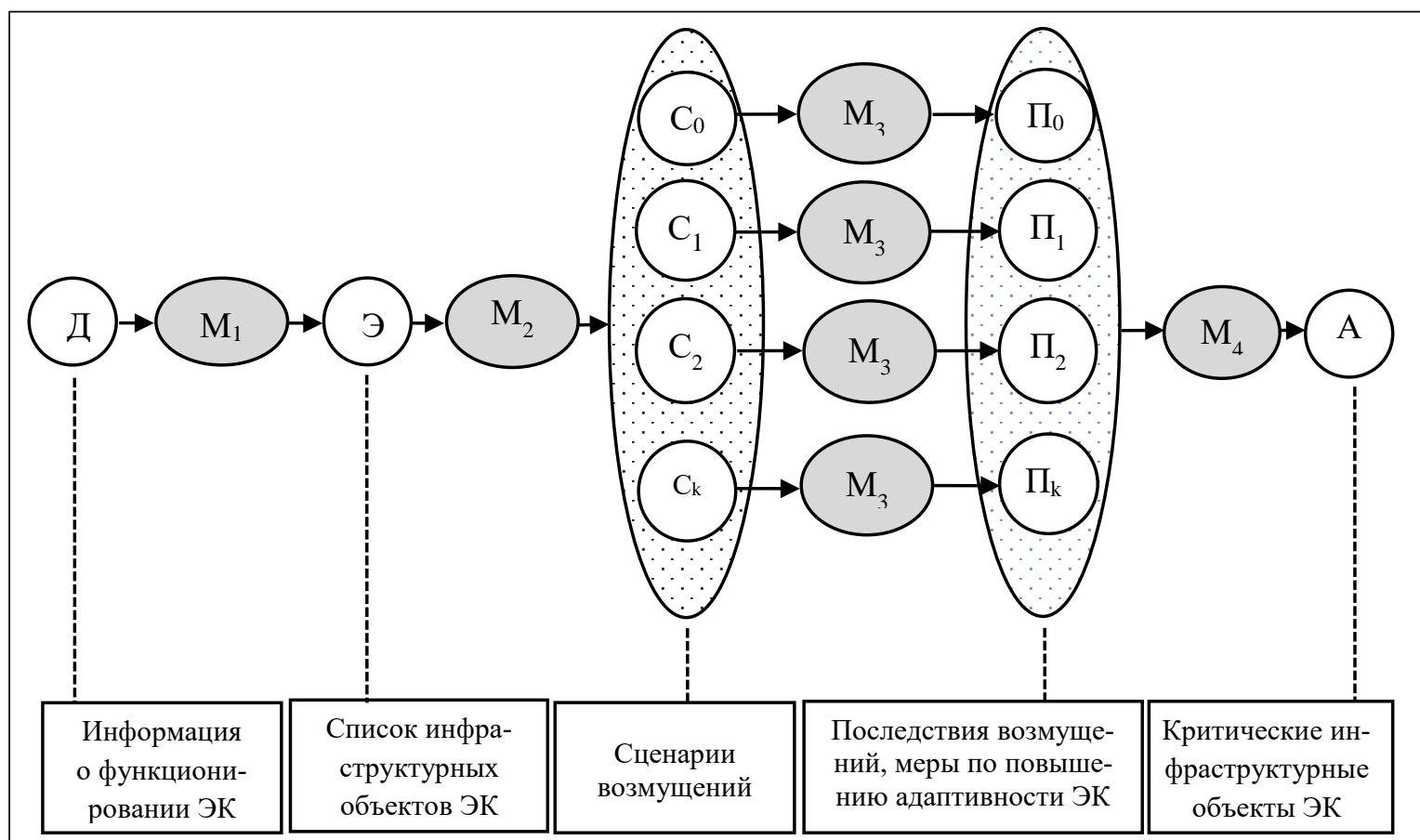

Рис. 3. Основные программные модули пакета для поиска критических элементов ЭК

Fig. 3. The main software modules of the packet for searching for energy complex critical elements 
осуществляются под управлением инструментария Orlando Tools [21]. Он предоставляет пользователям графический интерфейс для описания модулей пакета и схем решения задач предметной области, которые могут формулироваться как в процедурной, так и в декларативной форме. В последнем случае производится автоматический синтез схемы решения задачи. Схема, сформированная на основе ее процедурной постановки, может включать управляющие конструкции ветвления, цикла и рекурсии. Важной особенностью Orlando Tools является комплексирование разрабатываемых пакетов.

Структура типовой БД для моделирования функционирования взаимосвязанных СЭ в условиях возмущений подробно описана в [22]. Она состоит из следующих групп таблиц: справочники кодов инфраструктурных объектов ЭК, энергических технологий и ресурсов, исходные данные, описывающие территориально-производственную структуру СЭ, составляющих ЭК, и параметры их функционирования, а также правила для формирования моделей СЭ, входящих в состав ЭК.

\section{Заключение}

Представленный в статье подход к моделированию функционирования компонент взаи- мосвязанных СЭ в условиях возмущений описывает ЭК как метасистему взаимосвязанных отраслевых систем. Модель каждой системы разбивается на две части: структурную и функциональную. Структурная часть представляет топологию СЭ в виде сети или графа, который может быть ориентированным. Функциональная часть описывает распределение потока энергоресурсов по СЭ и ее реакцию на воздействие возмущения. Модель метасистемы получается путем объединения структурных и функциональных частей отдельных систем на основе предопределенных взаимосвязей между ними. Подход является универсальным по отношению к различным классам возмущений и ЭК разных уровней территориальной иерархии.

Применение подхода продемонстрировано на разработке модели энергетики страны, которая может использоваться для оценки адаптивности ЭК, например, для глобального или пространственного анализа уязвимости, поиска критических элементов.

Универсальный характер предлагаемого подхода поддерживается и на уровне его реализации с помощью средств метапрограммирования языка $\mathrm{C}++$. В качестве примера приводится описание распределенного пакета прикладных программ для поиска критических элементов ЭК.

Работа выполнена при финансовой поддержке Минобрнауки России в рамках Госзадания № FWEU2021-0003, а также РФФИ, проект № 15-07-07412-а. Структура базы данных для моделирования функиионирования взаимосвязанных систем энергетики разработана при финансовой поддержке РФФИ и Правительства Иркутской области, проект № 20-47-380002-р_а.

\section{Лuтература}

1. Voropai N., Rehtanz C. Flexibility and resiliency of electric power systems: Analysis of definitions and content. EPJ Web of Conferences, 2019, vol. 217, art. 01018. DOI: 10.1051/epjconf/201921701018.

2. Zio E. The future of risk assessment. Reliability Engineering and System Safety, 2018, vol. 177, pp. 176-190. DOI: 10.1016/j.ress.2018.04.020.

3. Zio E. Challenges in the vulnerability and risk analysis of critical infrastructures. Reliability Engineering and System Safety, 2016, vol. 152, pp. 137-150. DOI: 10.1016/j.ress.2016.02.009.

4. Espinoza S., Panteli M., Mancarella P., Rudnick H. Multi-phase assessment and adaptation of power systems resilience to natural hazards. Electric Power Systems Research, 2016, vol. 136, pp. 352-361. DOI: 10.1016/j.epsr.2016.03.019.

5. Carlson J.L., Haffenden R.A., Bassett G.W., Buehring W.A. et al. Resilience: Theory and application. ANL, 2012, ANL/DIS-12-1. URL: https://publications.anl.gov/anlpubs/2012/02/72218.pdf (дата обращения: 14.03.2021). DOI: $10.2172 / 1044521$.

6. Hosseini S., Barker K., Ramirez-Marquez J.E. A review of definitions and measures of system resilience. Reliability Engineering and System Safety, 2016, vol. 145, pp. 47-61. DOI: 10.1016/j.ress.2015.08.006.

7. Bie Z., Lin Y., Li G., Li F. Battling the extreme: A study on the power system resilience. Proc. IEEE, 2017, vol. 105, no. 7, pp. 1253-1266. DOI: 10.1109/JPROC.2017.2679040.

8. Еделев А.В., Феоктистов А.Г. Выбор подхода к моделированию взаимосвязанных критических инфраструктур // Современные проблемы и перспективные направления инновационного развития 
науки: тр. Междунар. науч.-практич. конф. 2021. С. 25-30. URL: https://aeterna-ufa.ru/sbornik/NK345.pdf (дата обращения: 14.03.2021).

9. Johansson J., Hassel H. Modelling, simulation and vulnerability analysis of interdependent technical infrastructures. In: Springer Series in Reliability Engineering, 2012, pp. 49-66. DOI: 10.1007/978-1-44714661-2_5.

10. Svegrup L., Johansson J., Hassel H. Integration of critical infrastructure and societal consequence models: impact on Swedish power system mitigation decisions. Risk Analysis, 2019, vol. 39, no. 9, pp. 1970-1996. DOI: $10.1111 /$ risa.13272.

11. Johansson J., Hassel H., Cedergren A. Vulnerability analysis of interdependent critical infrastructures: case study of the Swedish railway system. International Journal of Critical Infrastructures, 2011, vol. 7, no. 4, pp. 289-316. DOI: 10.1504/IJCIS.2011.045065.

12. Еделев А.В., Сендеров С.М., Береснева Н.М., Сидоров И.А., Феоктистов А.Г. Распределенная вычислительная среда для анализа уязвимости критических инфраструктур в энергетике // Системы управления, связи и безопасности. 2018. № 3. С. 197-231. URL: https://sccs.intelgr.com/archive/201803/10-Edelev.pdf (дата обращения: 14.03.2021).

13. Johansson J., Hassel H. An approach for modelling interdependent infrastructures in the context of vulnerability analysis. Reliability Engineering and System Safety, 2010, vol. 95, no. 12, pp. 1335-1344. DOI: 10.1016/j.ress.2010.06.010.

14. Ouyang M., Tao F., Huang S., Xu M., Zhang C. Vulnerability mitigation of multiple spatially localized attacks on critical infrastructure systems. Computer-Aided Civil and Infrastructure Engineering, 2018, vol. 33, no. 7, pp. 585-601. DOI: 10.1111/mice.12374.

15. Ouyang M., Liu C., Wu S. Worst-case vulnerability assessment and mitigation model of urban utility tunnels. Reliability Engineering and System Safety, 2020, vol. 197, art. 106856. DOI: 10.1016/j.ress.2020. 106856.

16. Jonsson H., Johansson J., Johansson H. Identifying critical components in technical infrastructure networks. Proc. Institution of Mechanical Engineers. Part O: Journal of Risk and Reliability, 2008, vol. 222, no. 2, pp. 235-243. DOI: 10.1243/1748006XJRR138.

17. Санеев Б.Г., Соколов А.Д., Агафонов Г.В. и др. Методы и модели разработки региональных энергетических программ. Новосибирск: Наука, 2003. 140 с.

18. Санеев Б.Г., Беляев Л.С., Агафонов Г.В., Воробьева В.В., Головин А.П. и др. Восточный вектор энергетической стратегии России: современное состояние, взгляд в будущее. Новосибирск: Гео, 2011 $368 \mathrm{c}$.

19. Зоркальцев В.И. Методы прогнозирования и анализа эффективности функционирования системы топливоснабжения. М.: Наука, 1988. 144 с.

20. Edelev A.V., Sidorov I.A., Gorsky S.A., Feoktistov A.G. Large-scale analysis of the energy system vulnerability using an in-memory data grid. Proc. The Intern. Workshop on Information, Computation, and Control Systems for Distributed Environments, 2020, vol. 2638, pp. 89-98. DOI: 10.47350/ICCS-DE.2020.08.

21. Feoktistov A., Gorsky S., Sidorov I., Bychkov I., Tchernykh A., Edelev A. Collaborative development and use of scientific applications in Orlando tools: Integration, delivery, and deployment. Communications in Computer and Information Science, 2020, vol. 1087, pp. 18-32. DOI: 10.1007/978-3-030-41005-6_2.

22. Еделев А.В., Феоктистов А.Г. База данных для моделирования автономных энергетических комплексов // Техническая и технологическая модернизация России. Проблемы, приоритеты, перспективы: тр. Междунар. науч.-практич. конф. Казань: Изд-во АЭТЕРНА, 2021. С. 3-9. URL: https://aeternaufa.ru/sbornik/NK-TT-137.pdf (дата обращения: 20.03.2021).

\section{An approach to modeling the functioning of interdependent energy systems under disturbances and its software support}

A.V. Edelev ${ }^{1}$, Ph.D. (Engineering), Senior Researcher, flower@isem.irk.ru N.M. Beresneva ${ }^{1}$, Ph.D. (Engineering), Research Associate, beresneva@isem.irk.ru

${ }^{1}$ Melentiev Energy Systems Institute SB RAS, Irkutsk, 664033, Russian Federation

Abstract. The purpose of the study is to develop an approach to modeling the functioning of interconnected energy systems under disturbance conditions, which has a universal character in relation to different classes of disturbances and energy complexes of different levels of the territorial hierarchy. 
The relevance of the work is determined by the need to ensure the energy sector sustainable functioning affected by various disturbances ranging from high fluctuations in energy prices and worsening weather conditions up to large-scale natural disasters and man-made disasters.

The novelty of the approach in comparison with others is its focus on using high-performance computing to speed up calculations and to divide the energy infrastructure model into structural and functional parts.

The structural part represents the infrastructure topology in the form of a network. The functional part describes the distribution of energy flows across the infrastructure and its response to the impact of disturbances. The division of the energy infrastructure model into structural and functional parts is a fundamental principle in modeling not only energy systems, but also energy complexes, which are considered as metasystems of interconnected energy systems in the proposed approach.

The energy complex model is built by combining the structural and functional parts of the models of individual energy systems based on predetermined relationships between them. The universal nature of the proposed approach is also supported by its software implementation using the meta-programming tools of the $\mathrm{C}++$ language, which significantly saves time when creating software packages designed to assess the adaptability of energy complexes.

The paper demonstrates the application of the approach on the development of a country's energy model that is used in a distributed package of applied programs for global or spatial analysis of vulnerability, search for critical elements of the energy complex.

Keywords: energy system, energy sector, adaptability, vulnerability, distributed computing environment, application package.

Acknowledgements. This work was financially supported by the Ministry of Education and Science of the Russian Federation in the framework of State Assignment no. FWEU-2021-0003 and RFBR in the framework of the scientific project no. 15-07-07412a. Developing the database structure for modeling the operation of interconnected energy systems was funded by the RFBR and Government of Irkutsk Region, project no. 20-47-380002.

\section{References}

1. Voropai N., Rehtanz C. Flexibility and resiliency of electric power systems: Analysis of definitions and content. EPJ Web of Conferences, 2019, vol. 217, art. 01018. DOI: 10.1051/epjconf/201921701018.

2. Zio E. The future of risk assessment. Reliability Engineering and System Safety, 2018, vol. 177, pp. 176-190. DOI: 10.1016/j.ress.2018.04.020.

3. Zio E. Challenges in the vulnerability and risk analysis of critical infrastructures. Reliability Engineering and System Safety, 2016, vol. 152, pp. 137-150. DOI: 10.1016/j.ress.2016.02.009.

4. Espinoza S., Panteli M., Mancarella P., Rudnick H. Multi-phase assessment and adaptation of power systems resilience to natural hazards. Electric Power Systems Research, 2016, vol. 136, pp. 352-361. DOI: 10.1016/j.epsr.2016.03.019.

5. Carlson J.L., Haffenden R.A., Bassett G.W., Buehring W.A. et al. Resilience: Theory and application. ANL, 2012, ANL/DIS-12-1. Available at: https://publications.anl.gov/anlpubs/2012/02/72218.pdf (accessed March 14, 2021). DOI: 10.2172/1044521.

6. Hosseini S., Barker K., Ramirez-Marquez J.E. A review of definitions and measures of system resilience. Reliability Engineering and System Safety, 2016, vol. 145, pp. 47-61. DOI: 10.1016/j.ress.2015.08.006.

7. Bie Z., Lin Y., Li G., Li F. Battling the extreme: A study on the power system resilience. Proc. IEEE, 2017, vol. 105, no. 7, pp. 1253-1266. DOI: 10.1109/JPROC.2017.2679040.

8. Edelev A.V., Feoktistov A.G. Choosing an approach to modeling interconnected critical infrastructures. Proc. Intern. Sciю and Pract. Conf. Modern Problems and Promising Directions of Science Innovative Development, 2021, pp. 25-30. Available at: https://aeterna-ufa.ru/sbornik/NK-345.pdf (accessed March 14, 2021) (in Russ.).

9. Johansson J., Hassel H. Modelling, simulation and vulnerability analysis of interdependent technical infrastructures. In: Springer Series in Reliability Engineering, 2012, pp. 49-66. DOI: 10.1007/978-1-44714661-2_5.

10. Svegrup L., Johansson J., Hassel H. Integration of critical infrastructure and societal consequence models: impact on Swedish power system mitigation decisions. Risk Analysis, 2019, vol. 39, no. 9, pp. 1970-1996. DOI: $10.1111 /$ risa.13272.

11. Johansson J., Hassel H., Cedergren A. Vulnerability analysis of interdependent critical infrastructures: case study of the Swedish railway system. International Journal of Critical Infrastructures, 2011, vol. 7, no. 4, pp. 289-316. DOI: 10.1504/IJCIS.2011.045065. 
12. Edelev A.V., Senderov S.M., Beresneva N.M., Sidorov I.A., Feoktistov A.G. Distributed computing environment for an analysis of the vulnerability of critical infrastructures in energy sector. Systems of Control, Communication and Security, 2018, no. 3, pp. 197-231. Available at: https://sccs.intelgr.com/archive/201803/10-Edelev.pdf (accessed March 14, 2021) (in Russ.).

13. Johansson J., Hassel H. An approach for modelling interdependent infrastructures in the context of vulnerability analysis. Reliability Engineering and System Safety, 2010, vol. 95, no. 12, pp. 1335-1344. DOI: 10.1016/j.ress.2010.06.010.

14. Ouyang M., Tao F., Huang S., Xu M., Zhang C. Vulnerability mitigation of multiple spatially localized attacks on critical infrastructure systems. Computer-Aided Civil and Infrastructure Engineering, 2018, vol. 33, no. 7, pp. 585-601. DOI: $10.1111 /$ mice. 12374 .

15. Ouyang M., Liu C., Wu S. Worst-case vulnerability assessment and mitigation model of urban utility tunnels. Reliability Engineering and System Safety, 2020, vol. 197, art. 106856. DOI: 10.1016/j.ress.2020. 106856.

16. Jonsson H., Johansson J., Johansson H. Identifying critical components in technical infrastructure networks. Proc. Institution of Mechanical Engineers. Part O: Journal of Risk and Reliability, 2008, vol. 222, no. 2, pp. 235-243. DOI: 10.1243/1748006XJRR138.

17. Saneev B.G., Sokolov A.D., Agafonov G.V. et al. Methods and Models for Developing Regional Energy Programs. Novosibirsk, 2003, 140 p. (in Russ.).

18. Saneev B.G., Belyaev L.S., Agafonov G.V., Vorobyova V.V., Golovin A.P. et al. The Eastern Vector of Russia's Energy Strategy: State of the Art and Prospects. Novosibirsk, 2011, 368 p. (in Russ.).

19. Zorkaltsev V.I. The Methods for Forecasting and Analysis of the Fuel Supply System Efficiency. Moscow, 1988. 144 p. (in Russ.).

20. Edelev A.V., Sidorov I.A., Gorsky S.A., Feoktistov A.G. Large-scale analysis of the energy system vulnerability using an in-memory data grid. Proc. The Intern. Workshop on Information, Computation, and Control Systems for Distributed Environments, 2020, vol. 2638, pp. 89-98. DOI: 10.47350/ICCS-DE.2020.08.

21. Feoktistov A., Gorsky S., Sidorov I., Bychkov I., Tchernykh A., Edelev A. Collaborative development and use of scientific applications in Orlando tools: Integration, delivery, and deployment. Communications in Computer and Information Science, 2020, vol. 1087, pp. 18-32. DOI: 10.1007/978-3-030-41005-6_2.

22. Edelev A.V., Feoktistov A.G. A database for modeling autonomous energy complexes. Proc. Intern. Sci. and Pract. Conf. Technical and Technological Modernization of Russia. Problems, Priorities, Prospects, 2021, pp. 3-9. Available at: https://aeterna-ufa.ru/sbornik/NK-TT-137.pdf (accessed March 20, 2021) (in Russ.).

\section{Для цитирования}

Еделев А.В., Береснева Н.М. Подход к моделированию функционирования взаимосвязанных систем энергетики в условиях возмущений и его программная поддержка / / Программные продукты и системы. 2021. Т. 34. № 3. С. 409-419. DOI: 10.15827/0236-235X.135.409-419.

\section{For citation}

Edelev A.V., Beresneva N.M. An approach to modeling the functioning of interdependent energy systems under disturbances and its software support. Software \& Systems, 2021, vol. 34, no. 3, pp. 409-419 (in Russ.). DOI: 10.15827/0236-235X.135.409-419. 